\title{
On fractional and semi-soft handover in long term evolution(LTE) networks
}

\begin{abstract}
The fast pace of development of telecommunications technology and the challenges presented with the increasing number of users for any service, anywhere, anytime, access creates new problems with on the base stations capability and the handover/handoff (HO) techniques. To face these challenges much research have been made to come out with algorithms that can handle handover of user equipment (UE) while maintaining their respective quality of service (QoS). In this paper a hybrid $\mathrm{HO}$ algorithms called semi-soft handover (SSHO) and fractional soft handover (FSHO) in long term evolution (LTE) system are studied. The outage probability, the main metric to evaluate handover, is compared for different handover techniques.
\end{abstract}

Keyword: Handover/handoff (HO); Long term evolution (LTE); Semi-soft handover (SSHO); Fractional soft handover (FSHO); Site selection diversity transmission (SSDT) 